\title{
La transformación del proceso de enseñanza-aprendizaje del taller de diseño interdisciplinar: de lo presencial al trabajo colaborativo en línea. Nuevos retos académicos
}

María Elena Molina Ayala y Jaime Javier Loredo Zamarrón

\section{Resumen}

$\mathrm{E}$ 1 presente trabajo pretende compartir la experiencia de vida de estudiantes y profesores de la Facultad del Hábitat de la Universidad Autónoma de San Luis Potosí (UASLP), surgida a partir de la transformación en los procesos de enseñanza-aprendizaje, que, de manera repentina y forzados por una pandemia, pasaron de lo presencial a lo virtual en un taller de diseño que se lleva a cabo en el noveno semestre de las seis licenciaturas impartidas en la Facultad, el cual fundamenta su esencia en el trabajo colaborativo e interdisciplinar con responsabilidad social; y lo desarrolla en comunidades vulnerables, basando su proceso de diseño en un diagnóstico obtenido a partir de la convivencia y la relación entre la comunidad universitaria y la del lugar objeto del estudio.

Palabras clave: experiencia docente, aprendizaje en línea, COVID-19.

\section{El taller interdisciplinar en la Facultad del Hábitat}

El taller interdisciplinar es un espacio de reflexión y de intercambio de saberes e ideas entre profesores y estudiantes de noveno semestre de la Facultad del Hábitat de la UASLP, integrado por las disciplinas de Diseño urbano y del paisaje, Arquitectura, Edificación y administración de obras, Restauración de bienes materiales muebles, Diseño industrial y Diseño gráfico, que trabajamos de manera holística y sistémica en una comunidad.

Cada semestre que se imparte este taller se pretende ir mejorando el acercamiento con la comunidad con la que se trabaja, la participación de ésta en el diseño del espacio no es un proceso fijo, según Geilfus (2002), existen diferentes formas de participación, las cuales varían dependiendo del grado de involucramiento social, que van desde la pasividad casi completa, al control del propio proceso en el que las personas son actores del autodesarrollo. De acuerdo a García (2012), lo que determina realmente la participación de la gente es el grado de decisión que tienen en el proceso. En el caso del taller interdisciplinar, se trabaja de manera muy estrecha con la gente, considerándola en todo momento para el diseño de los proyectos, propiciando con ello la autonomía en su funcionamiento cuando son entregados. Generalmente se trabaja con comunidades que tienen altos índices de marginación o alguna peculiaridad que requiere de la colaboración de los estudiantes para su mejora, haciéndose proyectos integrales que van desde el diseño urbano hasta el diseño del objeto o de los elementos de orientación, considerando las características del lugar y su cultura, la economía, los aspectos políticos y sociales, los materiales y técnicas constructivas, el presupuesto con que se cuenta, la factibilidad y viabilidad del proyecto, así como el impacto social y ambiental que se llega a tener, pero, sobre todo, se considera a las personas, convirtiéndose en un trabajo del hombre para el hombre: un trabajo humano. 


\section{En vínculo con la comunidad}

A lo largo de los años, se han desarrollado proyectos en diferentes municipios del estado de San Luis Potosí, como Guadalcázar, Venado o Salinas, así como proyectos al interior de la ciudad como el Instituto Estatal de Ciegos (IEC); y El país de las maravillas, una escuela que está inserta en la Facultad de Psicología de la UASLP. En la imagen 1 se puede observar la cantidad de personas integradas e involucradas en los proyectos.

Imagen 1. Convivencia de profesores y estudiantes de la Facultad del Hábitat, Guadalcázar, San Luis Potosí

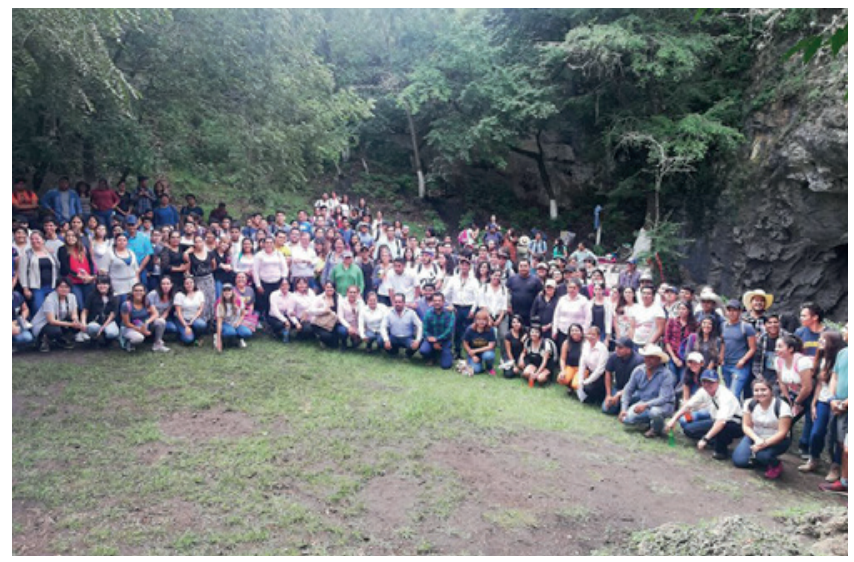

Fuente: fotografía de María Elena Molina Ayala, 2020.

Estos proyectos implican mucha cercanía y relación, no sólo con la comunidad, sino entre los estudiantes y los profesores. Las habilidades sociales, según Bilbao y Velasco (2014), son necesarias para el buen funcionamiento y armonía del grupo, tanto en lo referente al aprendizaje como a las relaciones entre los educandos; se requieren lazos fuertes para trabajar con las personas que posibiliten la realización de ejercicios de diseño participativo, entrevistas cara a cara o foto-etnografía, la comunidad nos abre la puerta de su casa y nos da información; es un proceso que dura un par de meses y nos permite conocer sus necesidades y ubicar a los estudiantes en un entorno real, muy similar al que enfrentarán en el ejercicio profesional.

A partir del diagnóstico asertivo, producto del acercamiento con la comunidad, se genera un concepto de diseño (Lupton, 2015) y se homologan métodos de trabajo entre las diferentes disciplinas para establecer el curso que tienen los proyectos a futuro, una vez establecido esto, se invita a los integrantes clave de la comunidad, así como a las autoridades del municipio, a revisar lo $\ll$ Se requieren lazos fuertes para trabajar

con las personas que posibiliten la realización

de ejercicios de diseño participativo. $\gg$ proyectado en su hábitat; en todo momento se considera la opinión de las personas, orientando los proyectos que se desarrollan hacia lo que les aporta mayor beneficio. Es un ejercicio que entiende al hábitat como sistema, porque escapa a nuestro control, ya que la mayor parte de la actividad se autoregula y desarrolla en niveles sobre entendidos o no declarados por los individuos, que tienen que ver con las normas y los valores, usos y costumbres de la comunidad que requieren un esfuerzo extra en nuestra comprensión del fenómeno.

Al iniciar el año 2020 se decidió trabajar en el municipio de Santa María del Río, SLP, un lugar turístico y pintoresco con altos índices de migración y marginación. De manera particular, los estudiantes estaban muy emocionados porque han tenido conocimiento previo de los ejercicios de vinculación realizados por otras generaciones de jóvenes con el taller interdisciplinar, de ahí que había mucha motivación por trabajar en la comunidad.

En el primer acercamiento participamos 120 estudiantes junto con 20 compañeros profesores, quienes fuimos recibidos con mucho agrado tanto por la comunidad como por las autoridades que nos presentaron el municipio. Nos ofrecieron una comida en la que hubo intercambio de ideas y presentación de sus necesidades.

Imagen 2. Clase virtual del taller interdisciplinar.

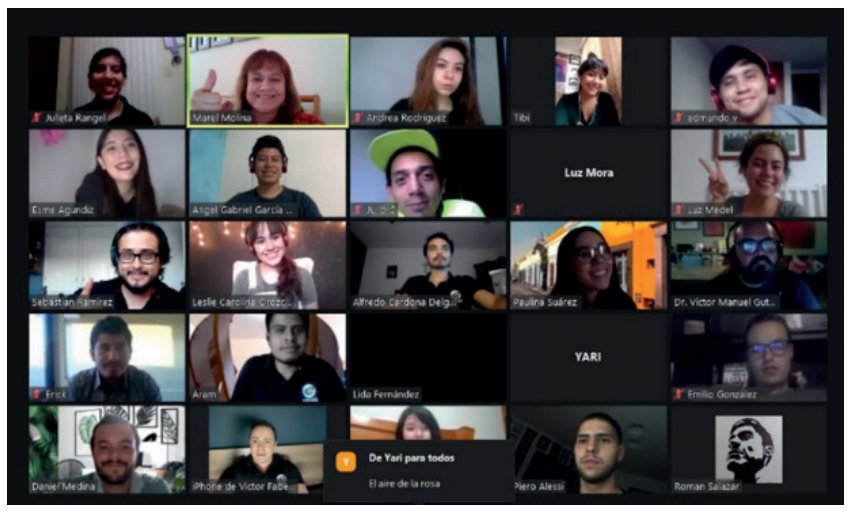

Fuente: Fotografía de María Elena Molina Ayala, 2020.

Estos acercamientos tienen muchas ventajas, pues permiten a los estudiantes comprender la realidad con la que se van a enfrentar al ejercer la profesión y generar propuestas pertinentes a la necesidad social, así como vin- 
cularse con el entorno, dejando de lado el diseño basado sólo en supuestos teóricos para confrontarse a realidades concretas y fortalecer el sentido de comunidad. También les enseña a trabajar de manera colaborativa e interdisciplinar, les hace darse cuenta de que se pueden fortalecer a partir de los saberes de las demás disciplinas, los estudiantes después de pasar por este proceso se convierten en individuos que entienden la importancia del trabajo colectivo (Posada, 2004).

\section{Del proceso de enseñanza-aprendizaje presencial al trabajo colaborativo en línea}

El 17 de marzo de 2020 se recibe, por parte de las autoridades de la UASLP, la indicación de que debíamos suspender labores presenciales debido a la contingencia de salud generada por el Covid-19 que afectaba a nivel global. Sin embargo, la Facultad del Hábitat en lo general y el taller interdisciplinar en lo particular, no se encontraban preparados para enfrentar el cambio de trasladar el trabajo de un modelo de aprendizaje presencial, a un modelo de aprendizaje virtual o híbrido, lo que representó un desafío para los docentes, los alumnos y la institución. Lo anterior resultó aún más complicado, ante la incertidumbre de saber cuándo se regresaría a la Facultad del Hábitat, donde en una primera instancia se consideraba la posibilidad de volver a clases presenciales al regreso de las vacaciones de semana santa; es decir, el lunes 20 de abril de 2020.

Una de las formas de comunicación complementaria con las que se contaba en ese momento con los estudiantes antes de la suspensión de clases era un grupo de WhatsApp, a través del cual se les proporcionaba material didáctico, lecturas de apoyo, se les entregaba un cronograma con las actividades y las formas de evaluar todo el curso, así como las rúbricas de evaluación y de coevaluación que se iban a considerar en el curso. Sin embargo, al suspenderse las clases presenciales, este grupo pasó a ser el principal mecanismo de comunicación del curso.

La ampliación del periodo de suspensión de clases, así como la incertidumbre de la fecha de retorno a las clases presenciales, motivó a considerar como un escenario plausible terminar el semestre en una modalidad virtual, por lo que el uso de WhatsApp tendría que regresar a ser un apoyo, pero no el elemento fundamental para continuar el curso a distancia. Tanto la Universidad Autónoma de San Luis Potosí como la Facultad del Hábitat contaban con experiencia en el uso de plataformas orientadas al aprendizaje. Existía la plataforma Didactic, administrada y ges- tionada por la Universidad; Teams de Microsoft con quien la universidad tiene convenio; y Schoology. Estas tres plataformas eran las más reconocidas y utilizadas por los maestros, ya que de ellas se habían desarrollado diferentes capacitaciones entre el personal docente. Sin embargo, hasta este momento, su uso siempre había sido opcional y como un complemento a las clases presenciales.

Al tratar de reestructurar el curso para una modalidad virtual, se presentaron tres problemas principales: 1) los recursos, 2) las habilidades y cultura digital de alumnos y maestros, y 3) la adaptación de los contenidos y mecánicas de trabajo de lo presencial a lo virtual.

Como en todo proceso de transformación, hubo muchas dudas y temores. La decisión más certera fue, tal vez, tener una estrecha comunicación entre los docentes y los alumnos que era independiente de horarios de clase. Una de las restricciones que implicaba el uso de las plataformas para llegar a los estudiantes era que no todos tenían acceso a internet, sin embargo, todos tenían un celular y podían buscar la forma de acceder en algún momento, esto nos hizo pensar en el siguiente principio para hacer accesible la educación: si lográbamos llegar al más vulnerable, lograríamos llegar a todos, por lo que se decidió que el medio de comunicación y de llevar a cabo el curso iba a ser a través de WhatsApp y de la plataforma gratuita Zoom. Se programaron las clases, y tuvimos sesiones regulares en el horario en que estaba establecida la impartición del taller.

Nos enfrentamos a la dificultad de trabajar de manera colaborativa en línea, pues en lo que nos adaptábamos se perdía mucho la parte del diálogo interdisciplinar, pero fue un aspecto que con la práctica se fue dando; sin embargo, la comunicación con la comunidad para la revisión de los resultados no se logró. Un asunto que sí fue exitoso fue el desarrollo de la parte humana del taller, se llevaron a cabo, vía remota, actividades lúdicas y no se dejaron pasar fechas importantes para festejar, como el día del maestro o el día del estudiante.

\section{El rescate de la experiencia aprendida a manera de conclusión}

El taller interdisciplinar está sometido permanentemente a procesos de cambio, ya sea por el tipo de estudiantes y profesores que participan o por la diversidad de contextos en los que se desarrolla. Bajo este principio, lo primero que requeríamos era entender este nuevo proceso, organizarnos y adaptarnos. López y Sánchez (2000) señalan que el arranque de las grandes y auténticas transformaciones 
escapa a menudo a nuestro control y descansa con mucha frecuencia en sucesos imprevistos. A pesar de lo fácilmente que podemos constatar esta afirmación, nuestra mente se resiste a aceptar que algo tan importante para nuestras vidas, como son los mecanismos que regulan el cambio de todo cuanto nos rodea, no estén en nuestras manos o que tengamos tan sólo una relativa influencia sobre ellos. Una vez entendido que no teníamos control sobre la transformación en el proceso de enseñanza-aprendizaje, fue más fácil ajustarnos a la nueva condición.

Iniciar el proceso de transformación requirió que se estableciera un diagnóstico para tratar de definir ¿quiénes somos?, ¿con qué recursos contamos?, ¿cómo los utilizamos?, a fin de establecer una estrategia clara respecto hacia dónde íbamos.

Se definieron los recursos con que contaban profesores y estudiantes, tanto de equipo de cómputo e internet como de habilidades y uso de herramientas digitales. Se estableció un canal permanente de comunicación, no sólo entre profesores, sino entre estudiantes y profesores. Se conocieron, a través del diálogo, aquellos aspectos personales que podían incidir en el rendimiento de los estudiantes. Se respetó el horario de clase y se estableció un horario de trabajo abierto para las dudas que pudieran surgir al trabajar a distancia. Se hizo énfasis en que tan importante era el proceso como el resultado. Se pidió que los estudiantes tuvieran un lugar fijo y determinado para trabajar, que fuera cómodo, bien iluminado y ventilado. Se trabajó con un cronograma que establecía fechas de entrega, actividades a realizar, responsables de las actividades y porcentaje de valor en la evaluación; esto dio certeza del proceso a los estudiantes.
《 Cómo en todo proceso de transformación hubo muchas dudas y temores. La decisión más certera fue tal vez, tener una estrecha comunicación entre los docentes y los alumnos.

Los cambios que se establecieron en la dinámica de trabajo no fueron del todo asimilados de inmediato por alumnos y maestros, ya que hubo elementos que lo dificultaron, entre los que destacan la falta de autodisciplina para el trabajo a distancia, el trabajo y el tiempo libre no estaban claramente separados, la estructura rutinaria de los cursos en academia de formación profesional exige mucha capacidad de adaptación, pero poca creatividad en el plano de la autoorganización, como señala Berzbach (2014); además de ello, el estado de ánimo de todos tenía días buenos y días malos que influían en el avance del proyecto.

Todos tenemos habilidades distintas que debemos conocer y aprovechar para fortalecer. Constantemente debemos replantear procesos que no son unidireccionales y sí multifactoriales. En el trabajo colaborativo es importante establecer roles y tener siempre una comunicación constante, no solamente estudiantes-profesores, sino entre los mismos estudiantes y la comunidad a la que servimos. Es fundamental establecer de manera constante un intercambio de saberes, tenemos mucho que aprender de nuestros estudiantes y de nuestros compañeros profesores. Es necesario estar evaluando constantemente el aprendizaje y construyendo herramientas en función de las necesidades particulares de los grupos. También resulta de gran valor compartir las experiencias docentes para poder enriquecernos, los procesos de enseñanza-aprendizaje son una responsabilidad compartida, todos estamos del mismo lado mirando hacia el futuro.

\section{Fuentes de consulta}

Berzbach, F. (2014). Psicología para creativos. Trabajar como creativo. Barcelona, España: Gustavo Gili.

Bilbao, M. del C. y Velasco, P. (2014). Aprendizaje cooperativo-colaborativo. México: Trillas.

García, W. (2012). Arquitectura participativa: las formas de lo esencial. Revista de Arquitectura, 14, 4-11. Recuperado el 2 de febrero de 2020, en https://www.redalyc.org/pdf/1251/125125877002.pdf

Geilfus, F. (2002). 80 herramientas para el desarrollo participativo: diagnóstico, planificación, monitoreo y evaluación. San José, Costa Rica: Frans Geilfus.

López, J. y Sánchez, M. (2000). Acerca del cambio en los sistemas complejos. Disponible en: https://bit.ly/3emN1nU Lupton, E. (2015). Intuición, acción, creación- graphic design thinking. Barcelona, España: Gustavo Gili.

Posada, R. (2004). Formación superior basada en competencias, interdisciplinariedad y trabajo autónomo del estudiante. Revista Iberoamericana de Educación. Recuperado el 14 de mayo de 2020, en http://www.rieoei.org/ deloslectores/648Posada.PDF 